\title{
Out-of-equilibrium dynamics driven by localized time-dependent perturbations at quantum phase transitions
}

\author{
Andrea Pelissetto, ${ }^{1}$ Davide Rossini, ${ }^{2}$ and Ettore Vicari ${ }^{2}$ \\ ${ }^{1}$ Dipartimento di Fisica dell'Università di Roma “La Sapienza” and INFN, Sezione di Roma I, I-00185 Roma, Italy \\ ${ }^{2}$ Dipartimento di Fisica dell'Università di Pisa and INFN, Largo Pontecorvo 3, I-56127 Pisa, Italy
}

(Received 5 January 2018; revised manuscript received 28 February 2018; published 14 March 2018)

\begin{abstract}
We investigate the quantum dynamics of many-body systems subject to local (i.e., restricted to a limited space region) time-dependent perturbations. If the system crosses a quantum phase transition, an off-equilibrium behavior is observed, even for a very slow driving. We show that, close to the transition, time-dependent quantities obey scaling laws. In first-order transitions, the scaling behavior is universal, and some scaling functions can be computed exactly. For continuous transitions, the scaling laws are controlled by the standard critical exponents and by the renormalization-group dimension of the perturbation at the transition. Our protocol can be implemented in existing relatively small quantum simulators, paving the way for a quantitative probe of the universal offequilibrium scaling behavior, without the need to manipulate systems close to the thermodynamic limit.
\end{abstract}

DOI: 10.1103/PhysRevB.97.094414

\section{INTRODUCTION}

Quantum phase transitions (QPTs) are one of the most striking signatures of many-body collective behavior, tantalizing the attention of a large body of theorists and experimentalists working in condensed-matter and statistical physics [1]. The standard paradigm of a QPT foresees a drastic change in the structural properties of the system at zero temperature when a given parameter in the Hamiltonian is tuned across some critical value. Generally, the driving parameters are global homogeneous quantities coupled to the critical modes, such as the magnetic field in spin systems [2-5], the chemical potential in particle systems [6-8], etc. However, close to a first-order transition, where equilibrium low-energy properties are particularly sensitive to localized external fields and/or boundary conditions, QPTs may also be driven by local perturbations [9].

It is also tempting to study the dynamics across QPTs induced by time-dependent parameters. Under these conditions, the system is inevitably driven out of equilibrium, even when the time dependence is very slow, because large-scale modes are unable to equilibrate as the system changes phase. Offequilibrium phenomena, such as, for example, hysteresis and coarsening, Kibble-Zurek defect production, aging, etc., have been addressed in a variety of contexts, both experimentally and theoretically (see, e.g., Refs. [10-20] and references therein). These studies mostly focused on the effects of slow changes of global parameters across classical and quantum transitions. They have shown that time-dependent properties of systems evolving under such dynamics obey off-equilibrium scaling (OS) behaviors, depending on the universal static and dynamic exponents of the equilibrium transition [21-26].

Here we overcome this paradigm and consider quantum systems subject to a local (i.e., restricted to a limited space region) time-dependent driving. We investigate whether and how these perturbations bring the system out of equilibrium as it moves across the different phases, showing the emergence of a universal behavior, as observed in the case of a global driving.
Our analysis provides a very intuitive and simple framework enabling us to develop a general OS theory that applies both to first-order and continuous quantum transitions (FOQTs and CQTs, respectively). The beauty of this approach resides in the possibility to quantitatively test universal quantum behavior even in a relatively small setting [27], without the need of much larger sizes approaching the thermodynamic limit (as, e.g., for the Kibble-Zurek framework), which would limit the experimental control over the sample and prevent quantitative testing. In view of the recent groundbreaking advancements in the field of quantum simulation, these issues acquire specific relevance as a proposal for experiments with a minimal set of requirements: manipulating and controlling individual quantum objects, without the need for scalability [16].

To fix the ideas, we concentrate on the quantum Ising ring, a paradigmatic model that undergoes various FOQTs and CQTs, when varying its parameters [1]. We present analytical and numerical results for the off-equilibrium behaviors arising from slow time-dependent protocols associated with local perturbations at its quantum transitions. They support the general OS arguments developed in the paper.

The paper is organized as follows. In Sec. II we introduce the quantum Ising ring model, and we review its equilibrium behavior in the presence of local (constant) perturbations at the quantum transitions. In Sec. III we develop the OS theory for slow time-dependent protocols associated with local perturbations at FOQTs; the OS functions of the quantum Ising ring along the FOQT line are computed by a two-level approximation, which turns out to be asymptotically exact. In Sec. IV we extend our study of the effects of time-dependent local perturbations at the CQT of the quantum Ising ring, showing that they give rise to OS behaviors as well. In Sec. V we study the off-equilibrium dynamics at the magnet-to-kink transition arising when a local bond perturbation is tuned along the FOQT line of the quantum Ising ring. Finally, Sec. VI presents a summary and our conclusions. In the Appendix, we focus on the dynamic two-level reduction exploited to compute 
the OS functions of the quantum Ising ring along the FOQT line.

\section{THE QUANTUM ISING RING}

The quantum Ising Hamiltonian for a ring of $L$ sites is given by

$$
H=-\sum_{x=0}^{L-1}\left[J \sigma_{x}^{(3)} \sigma_{x+1}^{(3)}+g \sigma_{x}^{(1)}+h \sigma_{x}^{(3)}\right] .
$$

The spin- $1 / 2$ variables $\sigma \equiv\left(\sigma^{(1)}, \sigma^{(2)}, \sigma^{(3)}\right)$ are the usual Pauli matrices, and $\sigma_{L}=\sigma_{0}$. We assume $\hbar=1, J=1$, and $g>0$. At $g=1$ and $h=0$, the model undergoes a CQT belonging to the two-dimensional Ising universality class, separating a disordered phase $(g>1)$ from an ordered $(g<1)$ one. For any $g<1$, the field $h$ drives FOQTs, due to the crossing of the two lowest-energy states $|+\rangle$ and $|-\rangle$ for $h=0$. Correspondingly, the longitudinal magnetization

$$
M=\frac{1}{L} \sum_{x=0}^{L-1} M_{x}, \quad M_{x} \equiv\left\langle\sigma_{x}^{(3)}\right\rangle
$$

is discontinuous, i.e. [2],

$$
\lim _{h \rightarrow 0^{ \pm}} \lim _{L \rightarrow \infty} M= \pm m_{0}, \quad m_{0}=\left(1-g^{2}\right)^{1 / 8},
$$

and $\left\langle \pm\left|\sigma_{x}^{(3)}\right| \pm\right\rangle= \pm m_{0}$. In a finite system of size $L$, the lowest states are superpositions of $|+\rangle$ and $|-\rangle$, due to tunneling effects. For $h=0$, their energy difference $\Delta$ vanishes exponentially as $L$ increases, $\Delta \sim g^{L}$, while the differences $\Delta_{i} \equiv E_{i}-E_{0}$ for the higher excited states $(i>1)$ are finite for $L \rightarrow \infty$. In particular, for the quantum Ising ring (corresponding to a chain with periodic boundary conditions) [2,28],

$$
\Delta \equiv \Delta_{1}(L) \approx 2\left(\frac{1-g^{2}}{\pi L}\right)^{1 / 2} g^{L} .
$$

The difference $\Delta_{i}$ for the higher excited states $(i>1)$ remains finite for $L \rightarrow \infty$, at any value of $g \neq 1$. In particular

$$
\Delta_{2}(L)=4(1-g)+O\left(L^{-2}\right) .
$$

Conversely, for $g=1, \Delta_{2}(L)=\pi /(2 L)+O\left(L^{-2}\right)$.

In the following, we wish to analyze the quantum dynamics in the presence of a single-site perturbation, adding

$$
H_{s}(t)=-s(t) \sigma_{0}^{(3)}
$$

to the Hamiltonian (1) with $h=0$. The control parameter $s(t)$ plays the role of a longitudinal magnetic field acting on one site only.

Before discussing the effects of a time-dependent perturbation, it is useful to summarize the equilibrium properties of the model when $s(t)$ is constant, $s(t)=s$. In the disordered phase $(g<1)$, the impact of the single-site perturbation is expected to be limited, being restricted within a region of finite size $\xi$. Therefore, for large-scale bulk quantities, the perturbation only gives rise to $O(\xi / L)$ corrections in the large- $L$ limit.

Approaching the CQT, i.e., for $g \rightarrow 1^{+}$, the system develops long-distance correlations, and $\xi$ diverges as $\xi \sim(g-1)^{-v}$ with $v=1$. Around $g=1$, the interplay between $\xi$ and $L$ originates an equilibrium finite-size scaling (EFSS) behavior
[29,30]. The effects of the local perturbation are amplified by long-distance correlations. Although they do not alter the leading power-law behavior, scaling functions acquire a nontrivial $s$-dependence. Moreover, local quantities acquire a nontrivial $x$-dependence. For instance, the local magnetization $M_{x}$ is expected to scale as [31-36]

$$
M_{x}(L, g=1, s) \approx L^{-\beta / \nu} \mathcal{M}_{E}\left(x_{p} / L, \xi / L, s L^{y_{s}}\right),
$$

where $x_{p}=\min (x, L-x)$ is the distance along the ring, $\beta=1 / 8$ is the magnetization exponent [1], and $y_{s}=1 / 2$ is the scaling dimension associated with the single-site parameter $s$ $[34,35]$. Thus, the average magnetization behaves as

$$
M(L, g=1, s) \approx L^{-\beta / v} Q_{E}\left(s L^{y_{s}}\right) .
$$

Along the FOQT line $(g<1)$, the system is particularly sensitive to local defects and boundary fields [9,37]. Indeed, the single-site perturbation $H_{s}$ can control the bulk phase: as $s$ changes sign, the bulk magnetization $M$ switches from $-m_{0}$ to $m_{0}$. An EFSS behavior can be defined at FOQTs [37], analogously to CQTs. In the case at hand, the relevant scaling variable is

$$
\kappa=\frac{2 m_{0} s}{\Delta},
$$

where $\Delta$ is the gap for $s=0$, defined in Eq. (4), so that

$$
M(L, g, s) \approx m_{0} f_{E}(\kappa)
$$

for any $g<1$.

The EFSS functions can be obtained by performing a two-level truncation, keeping only the lowest levels $| \pm\rangle$. This approximation holds whenever the energy difference between two such states remains much smaller than those between the higher excited states and the ground state. This requires

$$
\frac{\Delta}{\Delta_{2}} \approx \frac{1}{2}\left[\frac{1+g}{(1-g) \pi L}\right]^{1 / 2} g^{L} \ll 1
$$

for $s=0$, and

$$
m_{0}|s| \ll \Delta_{2} \approx 4(1-g),
$$

where we used the asymptotic behaviors of $\Delta$ and $\Delta_{2}$ at $s=0$; cf. Eqs. (4) and (5). For generic values of $g$, Eq. (11) is already satisfied for moderately large sizes. For example, for $g=1 / 2$, $\Delta / \Delta_{2} \approx 0.0068$ for $L=5$ and $\Delta / \Delta_{2} \approx 0.00067$ for $L=8$. [37]

The Hamiltonian restricted to this subspace has the form

$$
H_{e}=\left(\begin{array}{cc}
\varepsilon-\beta & \delta e^{i \varphi} \\
\delta e^{-i \varphi} & \varepsilon+\beta
\end{array}\right),
$$

where $\beta=m_{0} s$ represents the perturbation induced by the local magnetic field $s$, and $\delta=\Delta / 2$ is a small parameter that vanishes for $L \rightarrow \infty$ and $s=0$, giving rise to a degenerate ground state. The phase $\varphi$ is irrelevant, thus we $\operatorname{can} \operatorname{set} \varphi=0$ (it can be absorbed in the definition of the states). The eigenstates of $H_{e}$ are $(0<\alpha \leqslant \pi / 2)$

$$
\begin{aligned}
& |0\rangle=\sin (\alpha / 2)|-\rangle+\cos (\alpha / 2)|+\rangle, \\
& |1\rangle=\cos (\alpha / 2)|-\rangle-\sin (\alpha / 2)|+\rangle,
\end{aligned}
$$


where

$$
\tan \alpha=\kappa^{-1}, \quad \kappa=\frac{\beta}{\delta}=\frac{2 m_{0} s}{\Delta} .
$$

Their energy difference is

$$
E_{1}-E_{0}=\Delta \sqrt{1+\kappa^{2}} .
$$

The magnetization is obtained by computing the expectation value of $\sigma^{(3)}$ on the ground state $|0\rangle$,

$$
f_{E}(\kappa)=\cos \alpha=\frac{\kappa}{\sqrt{1+\kappa^{2}}} .
$$

In the following, we discuss the quantum evolution of the Ising model (1) with $h=0$ in the presence of a local longitudinal field (6) obeying a linear time dependence,

$$
s(t)=c t,
$$

with time scale $t_{s} \sim c^{-1}$. The protocol starts at $t_{i}<0$ from the ground state at $s\left(t_{i}\right)=s_{i}<0$. Then, the quantum dynamics evolves up to $t=t_{f}>0, s\left(t_{f}\right)=s_{f}>0$, so that $s(t)$ crosses the critical value $s=0$. We compute observables, such as the magnetization and correlation functions, during the quantum evolution both along the FOQT line (Sec. III) and at its end point $g=1, h=1$, where an Ising CQT appears (Sec. IV). We stress that our protocol (19) is quite general, since arbitrary time dependences can be linearized around $s=0$. Below we comment more in depth on this point.

\section{OFF-EQUILIBRIUM FINITE-SIZE SCALING ALONG THE FOQT LINE}

\section{A. Off-equilibrium finite-size scaling}

In this section, we develop the off-equilibrium finite-size scaling (OFSS) theory for the quantum evolution arising from the time-dependent protocol associated with the local perturbation (6) along the FOQT line. For this purpose, we must identify the relevant scaling variables. Since EFSS should be recovered in the appropriate limit (defined below), one of them can be obtained from the equilibrium variable $\kappa=2 m_{0} s / \Delta(L)$ by replacing $s$ with $s(t)=c t$,

$$
\kappa \equiv \frac{2 m_{0} s(t)}{\Delta}=\frac{2 t}{\Delta t_{s}},
$$

where $t_{s} \equiv\left(m_{0} c\right)^{-1}$. A natural choice for a second OS variable is

$$
\theta \equiv t \Delta .
$$

We also define the related OS variables

$$
\begin{gathered}
v \equiv \Delta^{2} t_{s}=2 \theta / \kappa, \\
\tau \equiv t / \sqrt{t_{s}}=\operatorname{sign}(\theta) \sqrt{\kappa \theta / 2} .
\end{gathered}
$$

The OS limit is defined by $t, t_{s}, L \rightarrow \infty$, keeping the above OS variables fixed. In this limit, the magnetization is expected to show the OFSS behavior

$$
M\left(t, t_{s}, L\right) \approx m_{0} f_{O}(v, \kappa)=m_{0} F_{O}(v, \tau),
$$

where $\tau=\sqrt{v} \kappa / 2$. In the adiabatic limit $\left(t, t_{s} \rightarrow \infty\right.$ at fixed $L$ and $\left.t / t_{s}\right)$, EFSS must be recovered, so that

$$
f_{O}(v \rightarrow \infty, \kappa)=f_{E}(\kappa) .
$$

The OS behavior is expected to develop in a narrow range of $s(t) \approx 0$; indeed, since $\tau$ is kept fixed in the OS limit and $s(t) \sim \tau / \sqrt{t_{s}}$, the relevant interval of $s(t)$ decreases as $t_{s}$ increases. This implies that the OFSS behavior is independent of the initial and final values of $s$. The OS functions are universal, i.e., independent of $g$ along the FOQT line. The approach to OFSS is expected to be controlled by the ratio between $\Delta \sim e^{-c L}$ and $\Delta_{2}=O(1)$, cf. Eqs. (4) and (5), therefore in the case of model (1), to be exponentially fast. We stress that the above arguments are quite general and can be straightforwardly extended to any FOQT.

We may also consider a generic protocol characterized by the time scale $t_{s}$, i.e., $s(t)=S\left(t / t_{s}\right)$ with $S(0)=0$ and $S^{\prime}(0) \neq 0$. Since the OS limit is taken by keeping $\tau \equiv t / \sqrt{t_{s}}$ fixed, we can expand $S\left(t / t_{s}\right)$ in powers of $t / t_{s}=\tau / \sqrt{t_{s}}$ and only keep the leading term in the OS limit. Higher-order terms give $O\left(t_{s}^{-1 / 2}\right)=O(\Delta)$ contributions: they are exponentially suppressed with the system size.

\section{B. Two-level approximation}

The OS functions at the FOQTs of the Ising ring can be exactly computed. Remarkably, in a way similar to EFSS, in the long-time limit and for large systems, the scaling properties in a small interval around $s=0$ [more precisely, for $m_{0}|s(t)| \ll \Delta_{2}$ ] are well captured by a two-level truncation [37], which only takes into account the two nearly degenerate lowest-energy states. This is demonstrated in Appendix.

The effective evolution is determined by the Schrödinger equation

$$
i \partial_{t} \Psi(t)=H_{r}(t) \Psi(t),
$$

where $\Psi(t)$ is a combination of the states $|+\rangle$ and $|-\rangle$ only. The time-dependent Hamiltonian $H_{r}(t)$ can be determined by assuming that its matrix elements are analytic functions of $t / t_{s}$, and that the nonanalyticity only arises in the limit $L \rightarrow \infty$ when the two states become degenerate. Using the symmetry of the model, one can see that it is enough to consider

$$
H_{r}=-\frac{t}{t_{s}} \sigma^{(3)}+\frac{\Delta}{2} \sigma^{(1)},
$$

where $\Delta / 2=\delta$ is the same amplitude that enters the offdiagonal terms of Eq. (13).

The dynamics is analogous to that governing a two-level quantum-mechanical system in which the energy separation of the two levels is a function of time, which is known as the Landau-Zener (LZ) problem [38]. Therefore, the timedependent wave function for the quantum Ising ring can be derived from the LZ corresponding solutions [39]. We obtain

$$
\Psi(t)=C_{-}(v, \tau)|-\rangle+C_{+}(v, \tau)|+\rangle,
$$

where $C_{ \pm}$are functions of the scaling variables $v=t_{s} \Delta^{2}$ and $\tau=t / \sqrt{t_{s}}$. In particular, assuming $\Psi\left(\tau_{i}\right)=|-\rangle$ for $\tau_{i}=-\infty$, the OFSS function $F_{O}$ of the magnetization defined in Eq. (24) 


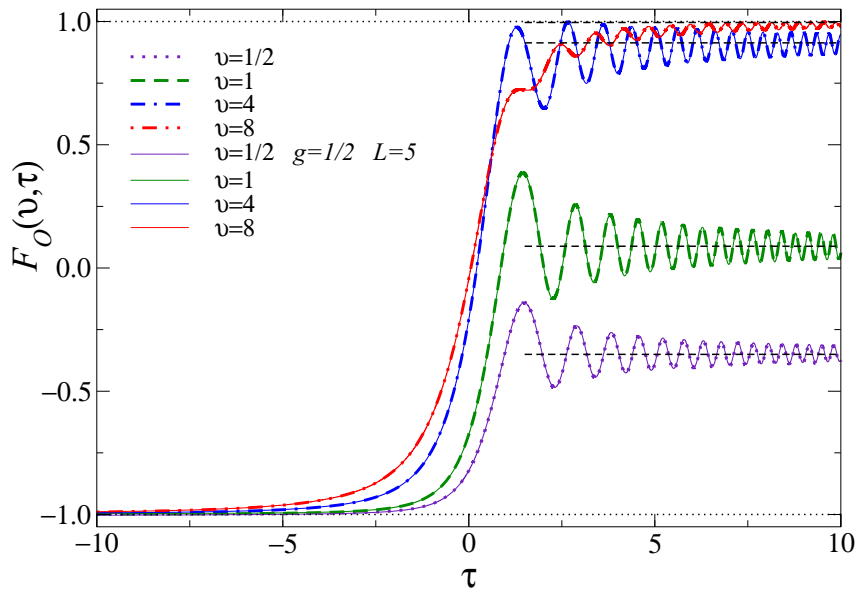

FIG. 1. The magnetization scaling function $F_{O}(v, \tau)$ reported in Eq. (29) for several values of $v$. We also plot the corresponding time evolution of the ratio $M / m_{0}$ for the Ising ring at $g=1 / 2$ and $L=5$ under the protocol (19). Differences are hardly visible. Note the oscillating behavior for $\tau>0$ around the asymptotic large- $\tau$ value [38] (short horizontal dashed lines), $F_{O}(v, \tau \rightarrow+\infty)=1-2 e^{-\pi v / 4}$; the amplitude of the oscillations slowly decreases with increasing $\tau$.

is given by

$$
\begin{aligned}
F_{O}(v, \tau) & =\left\langle\Psi(t)\left|\sigma^{(3)}\right| \Psi(t)\right\rangle \\
& =\left|C_{+}(v, \tau)\right|^{2}-\left|C_{-}(v, \tau)\right|^{2} \\
& =\frac{v}{4} e^{-\frac{\pi v}{16}}\left|D_{-1+i \frac{v}{8}}\left(\sqrt{2} e^{i \frac{3 \pi}{4}} \tau\right)\right|^{2}-1,
\end{aligned}
$$

where $D_{v}(z)$ is the parabolic cylinder function [40]. By replacing $\tau$ with $\sqrt{v} \kappa / 2$ in Eq. (29), we obtain the OS function $f_{O}(v, \kappa)$ defined in Eq. (24):

$$
f_{O}(v, \kappa)=F_{O}(v, \sqrt{v} \kappa / 2) \text {. }
$$

Note that the initial condition $\Psi\left(\tau_{i}=-\infty\right)=|-\rangle$ is consistent with the choice of the initial condition for the timedependent protocol (6), i.e., $\Psi\left(s_{i}<0\right)=|-\rangle$, because, when both $L$ and $t_{s}$ are large, any finite $s_{i}<0$ is in the adiabatic region, where the ground state is given by $\Psi\left(s_{i}\right) \approx|-\rangle$ with exponential precision. Indeed, a finite $s_{i}$ corresponds to $\kappa \rightarrow-\infty$ in the large- $L$ and $t_{s}$ limit keeping $\Delta^{2} t_{s}$ finite, and for $\kappa \rightarrow-\infty$ the ground state (14) is just given by $|-\rangle$.

Plots of the function $F_{O}(v, \tau)$ for some values of $v$ are shown in Fig. 1. We have also numerically computed the magnetization $M\left(t, t_{s}, L\right)$ for the quantum Ising ring [41]: the results displayed in Fig. 1 are in remarkable agreement with Eq. (29), even for small system sizes (we report data for $L=5$ ), reflecting the exponentially fast convergence to the asymptotic behavior. This validates the analytic derivation based on the two-level approximation.

Several notable limits of the solution (29) can be derived using the known properties of the parabolic cylinder function $[39,40] D_{v}(z)$. Concerning the $\tau$ dependence at fixed $v$, we obtain

$$
\begin{gathered}
F_{O}(v, \tau \rightarrow-\infty)=-1, \\
F_{O}(v, \tau=0)=-e^{-\pi v / 8}, \\
F_{O}(v, \tau \rightarrow+\infty)=1-2 e^{-\pi v / 4} .
\end{gathered}
$$

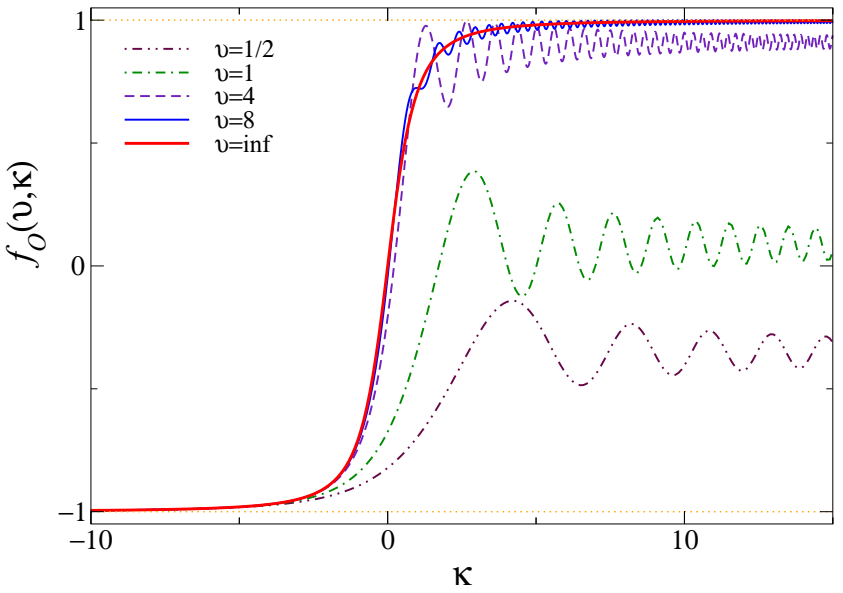

FIG. 2. The scaling function $f_{O}(v, \kappa)$ associated with the magnetization, cf. Eq. (30), for some values of $v$. In agreement with the OS arguments, it approaches the static limit $f_{E}(\kappa)=\kappa / \sqrt{1+\kappa^{2}}$ (red curve) when $v \rightarrow \infty$ keeping $\kappa$ fixed.

Note that the large- $\tau$ behavior is obtained by using the well-known KZ result for the transition probability from the ground state $|0\rangle$ to the excited state $|1\rangle$, which is given by $\left|C_{-}(v, \tau \rightarrow+\infty)\right|^{2}=e^{-\pi v / 4}$. The large- $v$ asymptotic behavior of $F_{O}(v, \tau)$ at fixed $\tau$ is

$$
F_{O}(v \gg 1, \tau) \approx \frac{2 \tau}{\sqrt{v+4 \tau^{2}}},
$$

so that $F_{O}(v, \tau)$ trivially vanishes for $v \rightarrow \infty$ and any finite $\tau$. The limit $v \rightarrow 0$ corresponds to the infinite-volume limit. We find

$$
F_{O}(v \rightarrow 0, \tau)=-1 .
$$

This reflects the effective decoupling of the states $| \pm\rangle$ of the Hamiltonian (27), which evolve independently in the infinite-volume limit.

In Fig. 2 we show some plots of the function $f_{O}(v, \kappa)$, obtained using Eq. (30). In agreement with the OS arguments, it approaches the static limit when $v \rightarrow \infty$ keeping $\kappa$ fixed. This is indeed confirmed by the solution (30). Replacing $\tau$ with $\sqrt{v} \kappa / 2$ in Eq. (34), we find

$$
f_{O}(v \rightarrow \infty, \kappa)=f_{E}(\kappa)=\frac{\kappa}{\sqrt{1+\kappa^{2}}},
$$

where $f_{E}(\kappa)$ is the EFSS function reported in Eq. (18).

The fluctuations of the magnetization can be characterized by its variance, given by

$$
\begin{aligned}
V_{M}(v, \tau) & =\left\langle\Psi(t)\left|\left(\sigma^{(3)}\right)^{2}\right| \Psi(t)\right\rangle-\left\langle\Psi(t)\left|\sigma^{(3)}\right| \Psi(t)\right\rangle^{2} \\
& =1-F_{O}(v, \tau)^{2} \leqslant 1 .
\end{aligned}
$$

Note that $V_{M}$ is generally of the same size of $F_{O}$.

These results show that the OFSS functions at the FOQTs of the Ising ring are well reproduced by the quantum dynamics of a two-level model, although the OS variables $\kappa, \theta$ are determined by the underlying many-body physics of the original model, which gives rise to the exponential dependence of the gap $\Delta(L)$. We stress that these conditions are only realized when the many-body system is tuned to the FOQT arising 
from a two-level crossing. An analogous behavior is expected in higher-dimensional quantum Ising systems.

Since the OFSS arguments leading to Eq. (24) apply to quite general FOQTs, our protocol can be seen as a viable proposal for a controlled quantum switch between the corresponding two states $|+\rangle$ and $|-\rangle$ in the symmetry-broken phase of a few-spin Ising-like chain, constituting an effective qubit. This would enhance its robustness with respect to other local codings (e.g., through the spin degree of freedom of a single atom or molecule). Switching from one to another state can be achieved by tuning a local longitudinal field, whose dynamical effects can be quantitatively controlled by universal scaling functions.

We finally mention that an analogous behavior is expected to emerge when the external magnetic field is spatially uniform, i.e., when one adds the magnetic term

$$
H_{h}=-h(t) \sum_{x=1}^{L} \sigma_{x}^{(3)}, \quad h(t)=a t
$$

instead of the local term (6). The protocol starts at $t_{i}<0$ from the ground state at $h_{i}=h\left(t_{i}\right)<0$, which is again given by $|-\rangle$ in the large- $L$ limit. Then, the system evolves up to a time $t_{f}>0$ corresponding to a finite $h_{f}>0$. The OS arguments apply here as well. One should only change the definition of $\kappa$, considering

$$
\kappa=\frac{2 m_{0} h(t) L}{\Delta} \equiv \frac{2 L t}{\Delta t_{s}},
$$

where we used the fact that the energy associated with the magnetic perturbation is $E_{h}=m_{0} h L$. The second scaling variable is again $\theta=\Delta t$, so that $v=\left(\Delta^{2} / L\right) t_{s}$. Using the fact that $\Delta=a_{g} L^{-1 / 2} e^{-c_{g} L}$, we may write the scaling variable corresponding to $\tau$, cf. Eq. (23), as $\tau \approx t t_{s}^{-1 / 2} \ln t_{s}$. Considerations based on the effective two-level model lead to the OFSS behavior (24) for the magnetization, with the same OS function $f_{O}(v, \kappa)$.

\section{OFF-EQUILIBRIUM DYNAMICS AT THE CONTINUOUS TRANSITION}

It is interesting to compare the behavior along the FOQT line with that occurring at its end point $g=1, h=0$, where a standard Ising CQT occurs. EFSS, Eq. (7), can be extended to the dynamic case using scaling arguments analogous to those used at the FOQT. The scaling variables are $\kappa=$ $\left(t / t_{s}\right) L^{y_{s}}$ with $y_{s}=1 / 2$ - this is the equilibrium scaling variable $s L^{y_{s}}$, in which we have simply replaced $s$ with $s(t)$ - and $\theta=t \Delta \sim t / L^{z}$ with $z=1$. We also define the related OS variables

$$
v \equiv t_{s} / L^{y_{s}+z}, \quad \tau \equiv t / t_{s}^{z /\left(z+y_{s}\right)} .
$$

Then, the local magnetization is expected to satisfy the OFSS equation

$$
M_{x}\left(L, t, t_{s}\right) \approx L^{-\beta / v} \mathcal{M}_{O}\left(x_{p} / L, v, \tau\right),
$$

so that its spatial average satisfies

$$
M\left(L, t, t_{S}\right) \approx L^{-\beta / v} Q_{O}(v, \tau) .
$$

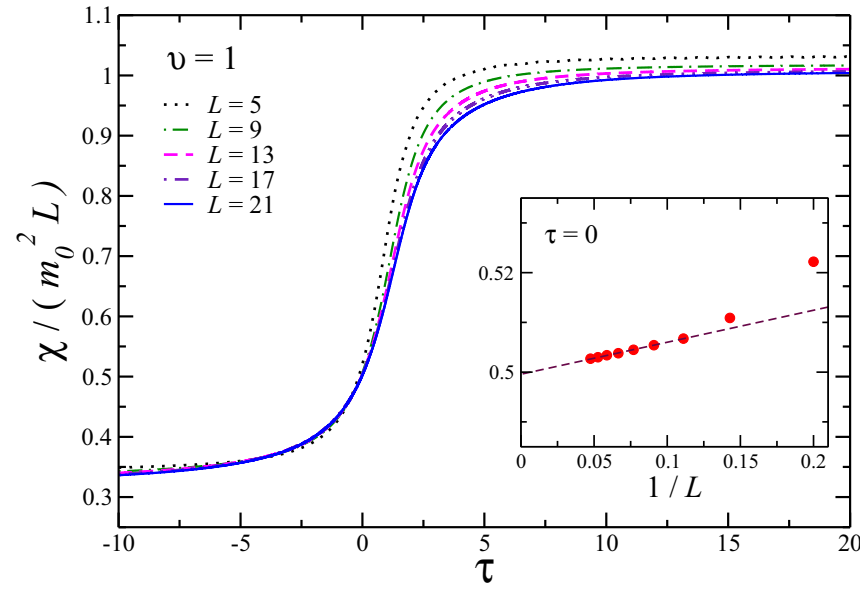

FIG. 3. Time-dependent magnetization at the CQT, in the presence of the single-site perturbation. We plot $L^{1 / 8} M$ vs $\tau=t / t_{s}^{2 / 3}$ for $v \equiv t_{s} / L^{3 / 2}=1$ and several values of $L$, as obtained by numerical calculations [41]. The curves clearly approach an asymptotic function with increasing $L$, confirming the existence of OFSS. In the inset, we show $L^{1 / 8} M$ for $t=\tau=0$ as a function of $1 / L$.

These OS behaviors are confirmed by numerics on moderately large systems [41], as displayed in Fig. 3 for $v=1$ (analogous results are obtained for other values of $v$ ). The inset shows that corrections decay as $1 / L$.

\section{OFF-EQUILIBRIUM DYNAMICS AT THE MAGNET-TO-KINK TRANSITIONS}

Other interesting examples of QPTs driven by a local perturbation arise when adding

$$
H_{b}(t)=b(t) \sigma_{\ell}^{(3)} \sigma_{\ell+1}^{(3)}, \quad \ell=\lfloor(L-1) / 2\rfloor
$$

to Hamiltonian (1) with $h=0$. In the static case, $b(t)=b$, such a term gives rise to CQTs when $g<1$, between two different quantum phases [9]: a magnet phase for $b<2$ and a kink phase for $b>2$.

In the magnet phase, the lowest states are superpositions of states with opposite magnetization $| \pm\rangle$ (neglecting local effects at the defect), and the gap is exponentially small $[9,42,43]$. In particular [9],

$$
\Delta \approx \frac{8 g}{1-g} w^{2} e^{-w L}, \quad w=\frac{1-g}{g}(2-b)
$$

for $b \rightarrow 2^{-}$. The large- $L$ two-point function,

$$
G\left(x_{1}, x_{2}\right) \equiv\left\langle\sigma_{x_{1}}^{(3)} \sigma_{x_{2}}^{(3)}\right\rangle,
$$

is trivially constant, i.e.,

$$
G_{r}\left(x_{1}, x_{2}\right) \equiv \frac{G\left(x_{1}, x_{2}\right)}{m_{0}^{2}} \rightarrow 1
$$

for $x_{1} \neq x_{2}$, keeping $X_{i} \equiv x_{i} / \ell$ fixed.

The behavior changes drastically when $b>2$, where the low-energy states are one-kink states, which behave as oneparticle states with $O\left(L^{-1}\right)$ momenta. The ground state and the first excited state are superpositions with definite parity of the lowest kink $|\downarrow \uparrow\rangle$ and antikink $|\uparrow \downarrow\rangle$ states. The gap behaves 
as [9]

$$
\Delta=\frac{8(b-1) g^{2}}{b(b-2)(1-g)^{2}} \frac{\pi^{2}}{L^{3}}+O\left(L^{-4}\right) .
$$

Moreover, the two-point function $G(x, y)$ behaves asymptotically as

$$
\frac{G\left(x_{1}, x_{2}\right)}{m_{0}^{2}}=1-\left|X_{1}-X_{2}\right|-\frac{\left|\sin \left(\pi X_{1}\right)-\sin \left(\pi X_{2}\right)\right|}{\pi},
$$

where $X_{i} \equiv x_{i} / \ell$.

The parameter $b$ turns out to drive a CQT at $b=b_{c}=2$, separating the magnet and kink phases [9], where the relevant scaling variable is

$$
\varepsilon_{s} \equiv \varepsilon L^{y_{\varepsilon}}, \quad \varepsilon \equiv b-2,
$$

with $y_{\varepsilon}=1$. In the scaling limit, the two-point function behaves as

$$
G\left(x_{1}, x_{2}\right) \approx m_{0}^{2} \mathcal{G}\left(X_{1}, X_{2} ; \varepsilon_{s}\right),
$$

which implies

$$
\chi \equiv \sum_{x} G(0, x)=m_{0}^{2} L f_{\chi}\left(\varepsilon_{s}\right) .
$$

Since $\Delta \sim L^{-2}$ at $b_{c}$, this CQT has $z=2$ as a dynamic exponent [9].

We should emphasize that this transition is driven by a local perturbation, contrary to the standard QPT paradigm, which requires a global tuning [1]. The key point is again associated with the underlying FOQT, which makes the system particularly sensitive to local defects.

We now study the off-equilibrium behavior arising when the system crosses the CQT. We consider a time-dependent bond variable $b(t)$ such that $b(0)=b_{c}=2$, obeying a linear time dependence:

$$
\varepsilon(t) \equiv b(t)-2=-t / t_{s} .
$$

We assume that the evolution starts at time $t_{i}<0$, so that $\varepsilon\left(t_{i}\right)=\varepsilon_{i}>0$ in the kink phase. Then, the system evolves up to $t=t_{f}>0$ corresponding to $\varepsilon\left(t_{f}\right)=\varepsilon_{f}<0$ in the magnet phase. Again we expect an off-equilibrium behavior when $\varepsilon(t)$ changes sign, which we describe using OS arguments analogous to those used in the case of the single-site perturbation.

Using OS arguments analogous to those of the previous section, we define the scaling variables

$$
\varepsilon_{t}=-L t / t_{s}, \quad \theta=t \Delta \sim t L^{-2},
$$

and also

$$
v=t_{s} L^{-3}, \quad \tau=t / t_{s}^{2 / 3} .
$$

In the limit $t, t_{s}, L \rightarrow \infty$ at fixed scaling variables, the observables are expected to show OFSS. For example, we expect

$$
G\left[x_{1}, x_{2} ; t, t_{s}, L\right] \approx m_{0}^{2} \mathcal{G}\left(X_{1}, X_{2} ; v, \tau\right)
$$

and also

$$
\chi \equiv \sum_{x} G\left(0, x ; t, t_{s}, L\right)=m_{0}^{2} L F_{\chi}(v, \tau) .
$$

Again, OFSS is confirmed by numerical computations [41], and corrections appear to decay as $1 / L$ (see Fig. 4).

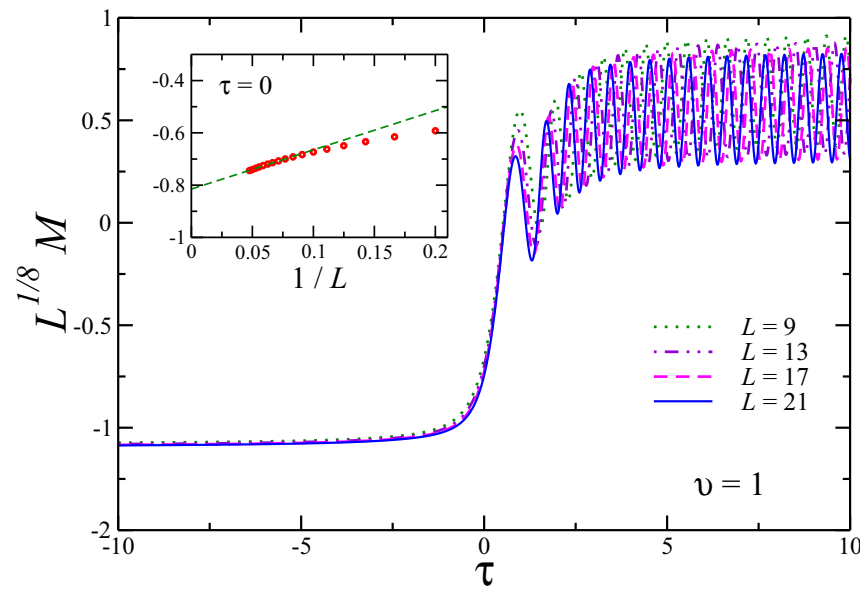

FIG. 4. Plot of $\chi /\left(m_{0}^{2} L\right)$ vs $\tau=t / t_{s}^{2 / 3}$ for $v \equiv t_{s} / L^{3}=1$ at the magnet-kink CQT for several values of $L$. We consider the Ising Hamiltonian at $h=0$ and $g=0.5$ with the bond perturbation (43). The curves are obtained by numerical calculations [41]. They clearly approach an asymptotic function with increasing $L$, confirming OFSS. The inset shows the same quantity at $\tau=0$ vs $1 / L$.

\section{SUMMARY AND CONCLUSIONS}

Summarizing, we have studied the effects of local timedependent perturbations of quantum many-body systems, focusing on phenomena induced by large time scales $t_{s}$. The off-equilibrium dynamics close to quantum transitions obeys general scaling laws. At a FOQT, the behavior can be parametrized by the scaling variables $v=\Delta^{2} t_{s}$ and $\tau=t / \sqrt{t_{s}}$. Some scaling functions can be predicted, for large system sizes, using a two-level Hamiltonian truncation. For CQTs, analogous scaling variables can be defined that are uniquely specified by the standard critical exponents and by the scaling dimension of the perturbation. Moreover, at FOQTs local variations of bond defects may lead to substantial changes of the bulk low-energy properties, leading to a dynamic behavior that admits an OS description as well. It is also possible to include the effect of a small finite temperature by adding the scaling variable $\rho=T / \Delta$.

The OS framework depicted here has been explicitly worked out in the quantum Ising model (1), but it is quite general. As a matter of fact, it can be extended to any FOQT and CQT, providing information on the possibility of controlling quantum phases, and their bulk low-energy properties, by local changes. Quite remarkably, the OFSS behavior can be observed for relatively small sizes: in some cases, a limited number of spins already displays asymptotic behavior (see, e.g., Fig. 1). Therefore, even systems of modest size may show definite signatures of the OS scaling laws derived in this work. In this respect, present-day quantum-simulation platforms have already demonstrated their capability to reproduce and control the dynamics of quantum Ising-like chains with $\sim 10$ spins. Ultracold atoms in optical lattices [4], trapped ions [5,44-48], and Rydberg atoms [49] seem to be the most promising candidates where the emerging universality properties of the quantum many-body physics discussed here can be tested with a minimal number of controllable objects. Furthermore, in quantum computing, some algorithms (notably the adiabatic 
ones) rely on a sufficiently large gap [50-52] and thus fail at FOQTs. The OS theory that we presented may clarify how this occurs in finite systems.

The OS arguments we developed can be extended to higher-dimensional systems, such as 2D and 3D quantum Ising systems at their FOQTs and CQTs, where novel features may arise depending on the various possible geometries of the defects. It is also tempting to generalize our framework to allow for dissipation, such as that induced by the coupling with an external bath in a Markovian framework [53-55]. The emergence of novel intriguing scenarios may be tested in near-future experiments based on cavity-QED technology with superconducting qubits [56-58].

\section{APPENDIX: TWO-LEVEL REDUCTION DURING THE DYNAMICS ALONG THE FOQT LINE}

Here we demonstrate that, similarly to EFSS, the dynamics in the OFSS limit can be determined by using a two-level truncation of the Hamiltonian. We consider a time-dependent Hamiltonian $H(t)$, and we assume that

$$
\frac{\partial H}{\partial t}=\frac{1}{t_{s}} A,
$$

where $A$ is independent of $t$. We recall that the dynamics starts at $t=t_{i}<0$ in one phase and ends for $t=t_{f}>0$ in the other phase. The transition point corresponds to $t=0$. For $t=t_{i}$ we require the system to be in the ground state of Hamiltonian $H\left(t_{i}\right)$, which we can identify with $|-\rangle$ in the large-volume limit.

To determine the dynamics, we should solve the evolution equation

$$
i \frac{\partial \Psi}{\partial t}=H(t) \Psi
$$

Let $\psi_{n}(t)$ and $E_{n}(t)$ be the orthonormalized eigenfunctions and eigenvalues of $H(t)$. Here $\psi_{0}$ is the ground state of the system and $\Delta_{1}(t)=E_{1}(t)-E_{0}(t)$. We expand $\Psi(t)$ as

$$
\Psi(t)=\sum_{n} c_{n}(t) \psi_{n}(t) e^{i \theta_{n}(t)}
$$

with

$$
\theta_{n}(t)=-\int_{t_{i}}^{t} E_{n}(s) d s
$$

For $t=t_{i}$ we have $\Psi=\psi_{0}\left(t_{i}\right)$ and therefore $c_{n}\left(t_{i}\right)=\delta_{n 0}$. Substitution of the expansion (A3) into Eq. (A2) gives

$$
\frac{d c_{n}}{d t}=-\sum_{k} c_{k}\left\langle\psi_{n} \mid \frac{\partial \psi_{k}}{\partial t}\right\rangle e^{i\left(\theta_{k}-\theta_{n}\right)},
$$

which must be solved with the boundary condition $c_{n}\left(t_{i}\right)=\delta_{n 0}$. Differentiating the eigenvalue equation $H(t) \psi_{n}=E_{n} \psi_{n}$ with respect to $t$, we obtain

$$
\left\langle\psi_{m}\left|\frac{\partial H}{\partial t}\right| \psi_{n}\right\rangle=\left(E_{n}-E_{m}\right)\left\langle\psi_{m} \mid \frac{\partial \psi_{n}}{\partial t}\right\rangle+\delta_{m n} \frac{\partial E_{n}}{\partial t} .
$$

Therefore, we obtain

$$
\begin{aligned}
\frac{d c_{n}}{d t}= & \sum_{k \neq n} c_{k} \frac{1}{t_{s}\left(E_{n}-E_{k}\right)}\left\langle\psi_{n}|A| \psi_{k}\right\rangle e^{i\left(\theta_{k}-\theta_{n}\right)} \\
& -c_{n}\left\langle\psi_{n} \mid \frac{\partial \psi_{n}}{\partial t}\right\rangle .
\end{aligned}
$$

If we just take the adiabatic limit $t_{s} \rightarrow \infty$, all cross terms can be neglected. Since $\psi_{n}$ is normalized, we can set

$$
\left\langle\psi_{n} \mid \frac{\partial \psi_{n}}{\partial t}\right\rangle=-i \phi_{n}(t),
$$

where $\phi_{n}(t)$ is a real function. Therefore, we have

$$
\frac{d c_{n}}{d t}=i c_{n} \phi_{n}(t)
$$

whose solution, with the given boundary conditions, is simply $c_{n}(t)=0$ for $n \geqslant 1$ and

$$
c_{0}(t)=\exp \left(i \int_{t_{i}}^{t} \phi_{0}\left(t^{\prime}\right) d t^{\prime}\right),
$$

which is merely the usual adiabatic theorem [59].

In our case, however, the previous approximation does not work as we are taking the limit at fixed

$$
t_{s}\left[E_{1}(t=0)-E_{0}(t=0)\right] \equiv t_{s} \Delta .
$$

Thus, we must proceed more carefully. First, we note that the differences $E_{n}(0)-E_{0}(0)$ and $E_{n}(0)-E_{1}(0)$ are strictly positive in the FSS limit for any $n \geqslant 2$. This implies that, in the FSS limit, $d c_{n} / d t$ for $n \geqslant 2$ depends only on $c_{k}$ with $k \geqslant 2$. Given that all $c_{n}$ with $n \geqslant 2$ vanish for $t=t_{i}$, we can conclude that we can set $c_{n}(t)=0$ for all $n \geqslant 2$. On the other hand, the coupling between the ground state and the first-excited state cannot be neglected. Hence, in the OFSS limit the dynamics can be determined by only considering two states, i.e., we can write

$$
\Psi(t)=c_{0}(t) \psi_{0}(t) e^{i \theta_{0}(t)}+c_{1}(t) \psi_{1}(t) e^{i \theta_{1}(t)},
$$

where $c_{0}(t)$ and $c_{1}(t)$ satisfy the coupled equations

$$
\begin{gathered}
\frac{d c_{0}}{d t}=i c_{0} \phi_{0}(t)-\frac{c_{1}}{t_{s} \Delta_{1}(t)}\left\langle\psi_{0}|A| \psi_{1}\right\rangle e^{i\left(\theta_{1}-\theta_{0}\right)}, \\
\frac{d c_{1}}{d t}=i c_{1} \phi_{1}(t)+\frac{c_{0}}{t_{s} \Delta_{1}(t)}\left\langle\psi_{1}|A| \psi_{0}\right\rangle e^{-i\left(\theta_{1}-\theta_{0}\right)} .
\end{gathered}
$$

Corrections are of order $1 / t_{s}$. Since $t_{s} \Delta$ is kept fixed in the OFSS limit, corrections decrease as $\Delta$, which is exponentially in the size of the system.

To make contact with the presentation in the paper, note that $\psi_{0}(t)$ and $\psi_{1}(t)$ are the first two lowest states of the model in the presence of a magnetic field. In the OFSS limit, as we have stressed at the beginning, these two states can be written as combinations of the magnetized states $|+\rangle$ and -$\rangle$. Therefore, we can obtain the correct dynamic scaling behavior by simply writing $\Psi(t)=e_{0}(t)|+\rangle+e_{1}(t)|-\rangle$ and considering the evolution restricted to the subspace spanned by these two states. Corrections are again expected to be exponentially small. 
[1] S. Sachdev, Quantum Phase Transitions (Cambridge University Press, Cambridge, 1999).

[2] P. Pfeuty, The one-dimensional Ising model with a transverse field, Ann. Phys. (N.Y.) 57, 79 (1970).

[3] R. Coldea, D. A. Tennant, E. M. Wheeler, E. Wawrzynska, D. Prabhakaran, M. Telling, K. Habicht, P. Smeibidl, and K. Kiefer, Quantum criticality in an Ising chain: Experimental evidence for emergent $E_{8}$ symmetry, Science 327, 177 (2010).

[4] J. Simon, W. S. Bakr, R. Ma, M. E. Tai, P. M. Preiss, and M. Greiner, Quantum simulation of antiferromagnetic spin chains in an optical lattice, Nature (London) 472, 307 (2011).

[5] R. Islam, E. E. Edwards, K. Kim, S. Korenblit, C. Noh, H. Carmichael, G.-D. Lin, L.-M. Duan, C.-C. Joseph Wang, J. K. Freericks, and C. Monroe, Onset of a quantum phase transition with a trapped ion quantum simulator, Nat. Commun. 2, 377 (2011).

[6] M. P. A. Fisher, P. B. Weichman, G. Grinstein, and D. S. Fisher, Boson localization and the superfluid-insulator transition, Phys. Rev. B 40, 546 (1989).

[7] M. Greiner, O. Mandel, T. Esslinger, T. W. Hänsch, and I. Bloch, Quantum phase transition from a superfluid to a Mott insulator in a gas of ultracold atoms, Nature (London) 415, 39 (2002).

[8] W. S. Bakr, A. Peng, M. E. Tai, R. Ma, J. Simon, J. I. Gillen, S. Fölling, L. Pollet, and M. Greiner, Probing the superfluid-toMott insulator transition at the single-atom level, Science 329, 547 (2010).

[9] M. Campostrini, A. Pelissetto, and E. Vicari, Quantum transitions driven by one-bond defects in quantum Ising rings, Phys. Rev. E 91, 042123 (2015); Quantum Ising chains with boundary fields, J. Stat. Mech. (2015) P11015.

[10] K. Binder, Theory of first-order phase transitions, Rep. Prog. Phys. 50, 783 (1987).

[11] A. J. Bray, Theory of phase-ordering kinetics, Adv. Phys. 43, 357 (1994).

[12] P. Calabrese and A. Gambassi, Ageing properties of critical systems, J. Phys. A 38, R133 (2005).

[13] D. Boyanovsky, J. J. de Vega, and D. J. Schwarz, Phase transitions in the early and the present universe, Annu. Rev. Nucl. Part. Sci. 56, 441 (2006).

[14] J. Dziarmaga, Dynamics of a quantum phase transition and relaxation to a steady state, Adv. Phys. 59, 1063 (2010).

[15] A. Polkovnikov, K. Sengupta, A. Silva, and M. Vengalattore, Colloquium: Nonequilibrium dynamics of closed interacting quantum systems, Rev. Mod. Phys. 83, 863 (2011).

[16] I. M. Georgescu, S. Ashhab, and F. Nori, Quantum simulation, Rev. Mod. Phys. 86, 153 (2014).

[17] G. Biroli, Slow relaxations and non-equilibrium dynamics in classical and quantum systems, in Strongly Interacting Quantum Systems Out of Equilibrium, Lect. Notes of the Les Houches Summer School, Vol. 99, edited by T. Giamarchi, A. J. Millis, O. Parcollet, H. Saleur, and L. F. Cugliandolo (Oxford University Press, Oxford, 2012).

[18] S. Ulm, S. J. Roßnagel, G. Jacob, C. Degünther, S. T. Dawkins, U. G. Poschinger, R. Nigmatullin, A. Retzker, M. B. Plenio, F. Schmidt-Kaler, and K. Singer, Observation of the Kibble-Zurek scaling law for defect formation in ion crystals, Nat. Commun. 4, 2290 (2013).

[19] G. Lamporesi, S. Donadello, S. Serafini, F. Dalfovo, and G. Ferrari, Spontaneous creation of Kibble-Zurek solitons in a Bose-Einstein condensate, Nat. Phys. 9, 656 (2013).
[20] N. Navon, A. L. Gaunt, R. P. Smith, and Z. Hadzibabic, Critical dynamics of spontaneous symmetry breaking in a homogeneous Bose gas, Science 347, 167 (2015).

[21] A. Polkovnikov, Universal adiabatic dynamics in the vicinity of a quantum critical point, Phys. Rev. B 72, 161201(R) (2005).

[22] W. H. Zurek, U. Dorner, and P. Zoller, Dynamics of a Quantum Phase Transition, Phys. Rev. Lett. 95, 105701 (2005).

[23] J. Dziarmaga, Dynamics of a Quantum Phase Transition: Exact Solution of the Quantum Ising Model, Phys. Rev. Lett. 95, 245701 (2005).

[24] S. Gong, F. Zhong, X. Huang, and S. Fan, Finite-time scaling via linear driving, New J. Phys. 12, 043036 (2010).

[25] A. Chandran, A. Erez, S. S. Gubser, and S. L. Sondhi, KibbleZurek problem: Universality and the scaling limit, Phys. Rev. B 86, 064304 (2012).

[26] A. Pelissetto and E. Vicari, Dynamic Off-Equilibrium Transition in Systems Slowly Driven Across Thermal First-Order Transitions, Phys. Rev. Lett. 118, 030602 (2017).

[27] J. Zhang, G. Pagano, P. W. Hess, A. Kyprianidis, P. Becker, H. Kaplan, A. V. Gorshkov, Z.-X. Gong, and C. Monroe, Observation of a many-body dynamical phase transition with a 53-qubit quantum simulator, Nature (London) 551, 601 (2017).

[28] G. G. Cabrera and R. Jullien, Universality of Finite-Size scaling: Role of The Boundary Conditions, Phys. Rev. Lett. 57, 393 (1986); Role of the boundary conditions in the finite-size Ising model, Phys. Rev. B 35, 7062 (1987).

[29] Finite Size Scaling and Numerical Simulation of Statistical Systems, edited by V. Privman (World Scientific, Singapore, 1990).

[30] M. Campostrini, A. Pelissetto and E. Vicari, Finite-size scaling at quantum transitions, Phys. Rev. B 89, 094516 (2014).

[31] K. Binder, Critical behavior at surfaces, in Phase Transitions and Critical Phenomena, edited by C. Domb and J. L. Lebowitz (Academic, London, 1983), Vol. 8, p. 1.

[32] M. N. Barber, Finite-size scaling, in Phase Transitions and Critical Phenomena, edited by C. Domb and J. L. Lebowitz (Academic, London, 1983), Vol. 8, p. 145.

[33] H. W. Diehl, Field-theoretic approach to critical behavior at surfaces, in Phase Transitions and Critical Phenomena, edited by C. Domb and J. L. Lebowitz (Academic, London, 1986), Vol. 10, p. 75.

[34] J. Cardy and D. Lewellen, Bulk and boundary operators in conformal field theory, Phys. Lett. B 259, 274 (1991).

[35] R. Chatterjee and A. Zamolodchikov, Local magnetization in critical Ising model with boundary magnetic field, Mod. Phys. Lett. A 09, 2227 (1994).

[36] E. Sela and A. K. Mitchell, Local magnetization in the boundary Ising chain at finite temperature, J. Stat. Mech. (2012) P04006.

[37] M. Campostrini, J. Nespolo, A. Pelissetto, and E. Vicari, Finitesize Scaling at First-Order Quantum Transitions, Phys. Rev. Lett. 113, 070402 (2014).

[38] C. Zener, Non-adiabatic crossing of energy levels, Proc. R. Soc. London, Ser. A 137, 696 (1932); L. Landau, On the theory of transfer of energy at collisions II, Phys. Z. Sowjetunion 2, 46 (1932).

[39] N. V. Vitanov and B. M. Garraway, Landau-Zener model: Effects of finite coupling dynamics, Phys. Rev. A 53, 4288 (1996); N. V. Vitanov, Transition times in the Landau-Zener model, ibid. 59, 988 (1999). 
[40] Handbook of Mathematica Functions, edited by M. Abramowitz and I. A. Stegun (Dover, New York, 1964).

[41] At the FOQTs, where a very high accuracy is needed in order to be able to discriminate exponential scalings, we calculated the magnetization by means of an exact diagonalization technique up to $L=7$. At CQTs, we exploited the fact that the Hamiltonian only involves nearest-neighbor couplings, and we simulated the dynamics of systems with up to 21 spins (the dimension of the Hilbert space is $\sim 2 \times 10^{6}$ ) by means of a Suzuki-Trotter decomposition of the unitary evolution operator as a product of local terms. The time has been discretized in small steps of $\Delta t=10^{-2}$.

[42] J. Zinn-Justin, Comment on Universality of Finite-Size Scaling: Role of the Boundary Conditions, Phys. Rev. Lett. 57, 3296 (1986).

[43] M. N. Barber and M. E. Cates, Effect of boundary conditions on the finite-size transverse Ising model, Phys. Rev. B 36, 2024 (1987).

[44] K. Kim, M.-S. Chang, S. Korenblit, R. Islam, E. E. Edwards, J. K. Freericks, G.-D. Lin, L.-M. Duan, and C. Monroe, Quantum simulation of frustrated Ising spins with trapped ions, Nature (London) 465, 590 (2010).

[45] E. E. Edwards, S. Korenblit, K. Kim, R. Islam, M.-S. Chang, J. K. Freericks, G.-D. Lin, L.-M. Duan, and C. Monroe, Quantum simulation and phase diagram of the transverse-field Ising model with three atomic spins, Phys. Rev. B 82, 060412(R) (2010).

[46] G.-D. Lin, C. Monroe, and L.-M. Duan, Sharp Phase Transitions in a Small Frustrated Network of Trapped ion Spins, Phys. Rev. Lett. 106, 230402 (2011).

[47] K. Kim, S. Korenblit, R. Islam, E. E. Edwards, M.-S. Chang, C. Noh, H. Carmichael, G.-D. Lin, L.-M. Duan, C. C. Joseph Wang, J. K. Freericks, and C. Monroe, Quantum simulation of the transverse Ising model with trapped ions, New J. Phys. 13, 105003 (2011).

[48] S. Debnath, N. M. Linke, C. Figgatt, K. A. Landsman, K. Wright, and C. Monroe, Demonstration of a small programmable quantum computer with atomic qubits, Nature (London) 536, 63 (2016).

[49] H. Labuhn, D. Barredo, S. Ravets, S. de Leseleuc, T. Macri, T. Lahaye, and A. Browaeys, Tunable two-dimensional arrays of single Rydberg atoms for realizing quantum Ising models, Nature (London) 534, 667 (2016).

[50] I. Bloch, Quantum coherence and entanglement with ultracold atoms in optical lattices, Nature (London) 453, 1016 (2008).

[51] A. P. Young, S. Knysh, and V. N. Smelyanskiy, First-Order Phase Transition in the Quantum Adiabatic Algorithm, Phys. Rev. Lett. 104, 020502 (2010).

[52] M. H. S. Amin and V. Choi, First-order quantum phase transition in adiabatic quantum computation, Phys. Rev. A 80, 062326 (2009).

[53] T. Albash, S. Boixo, D. A. Lidar, and P. Zanardi, Quantum adiabatic Markovian master equations, New J. Phys. 14, 123016 (2012).

[54] P. Nalbach, S. Vishveshwara, and A. A. Clerk, Quantum KibbleZurek physics in the presence of spatially correlated dissipation, Phys. Rev. B 92, 014306 (2015).

[55] M. Keck, S. Montangero, G. E. Santoro, R. Fazio, and D. Rossini, Dissipation in adiabatic quantum computers: Lessons from an exactly solvable model, New J. Phys. 19, 113029 (2017).

[56] M. J. Hartmann, F. G. S. L. Brandao, and M. B. Plenio, Effective Spin Systems in Coupled Microcavities, Phys. Rev. Lett. 99, 160501 (2007).

[57] A. A. Houck, H. E. Türeci, and J. Koch, On-chip quantum simulation with superconducting circuits, Nat. Phys. 8, 292 (2012).

[58] M. Fitzpatrick, N. M. Sundaresan, A. C. Y. Li, J. Koch, and A. A. Houck, Observation of a Dissipative Phase Transition in a One-Dimensional Circuit QED Lattice, Phys. Rev. X 7, 011016 (2017).

[59] A. Messiah, Quantum Mechanics (North Holland, Amsterdam, 1962), Vol. II 